\title{
Mejora de la toma de decisiones en ciclo de ventas del subsistema comercial de servicios en una empresa de IT
}

\author{
Improvement of sales decision making of the commercial services subsystem in an IT \\ company
}

\section{Melhoria da tomada de decisão de vendas do subsistema de serviços comerciais em uma empresa de TI}

\author{
Diego Armando Vanegas ${ }^{1}$ \\ Giovanny Mauricio Tarazona-Bermúdez $z^{2}$ \\ Luz Andrea Rodríguez-Rojas ${ }^{3}$ (D)
}

Recibido: agosto 2019

Aceptado: abril 2020

Para citar este artículo: Vanegas, D.A., Tarazona-Bermúdez, G.M., Rodríguez-Rojas, L.A. (2020). Mejora de la toma de decisiones en ciclo de ventas del subsistema comercial de servicios en una empresa de IT. Revista Científica, 38(2), 174-183. https://doi.org/10.14483/23448350.15241

\section{Resumen}

El alto volumen de datos, el tiempo de procesamiento y la visualización de información son problemas que hoy en día deben afrontar las organizaciones en el sector de tecnología, y en particular el subsistema comercial. En esta investigación se desarrolló un modelo para la toma de decisiones basado en la interacción de los criterios y las etapas del ciclo de ventas. Se usó una herramienta especializada en inteligencia de negocios que facilitó el manejo de grandes volúmenes de datos, su procesamiento y la visualización de la información. Dentro de los resultados conseguidos se encontró una significativa reducción en el tiempo de obtención de la información, pasando de horas a minutos.

Palabras clave: analítica de datos, analítica de negocios, ETL, ventas.

\begin{abstract} sales.
\end{abstract}

The high volume of data, the processing time and the visualization of information are problems that organizations in the technology sector, particularly the commercial subsystem, must face today. In this research, a model for decision-making based on the interaction of criteria and stages of the sales cycle was developed. A specialized business intelligence tool was used that facilitated the handling of large volumes of data, its processing, and the visualization of information. Among the results obtained, a significant reduction was found in the time of obtaining the information, it went from hours to minutes. Keywords: data analytics, business analytics, ETL,

1. Universidad Distrital Francisco José de Caldas. Bogotá, Colombia. diegovanegas@rocketmail.com

2. Universidad Distrital Francisco José de Caldas. Bogotá, Colombia. gtarazona@udistrital.edu.co

3. Universidad Distrital Francisco José de Caldas. Bogotá, Colombia. larodriguezr@udistrital.edu.co 


\section{Resumo}

O alto volume de dados, o tempo de processamento e a visualização de informações são problemas que as organizações do setor de tecnologia, particularmente o subsistema comercial, devem enfrentar hoje. Nesta pesquisa, foi desenvolvido um modelo de tomada de decisão baseado na interação de critérios e etapas do ciclo de vendas. Foi utilizada uma ferramenta especializada de business intelligence que facilitou o manuseio de grandes volumes de dados, seu processamento e a visualização de informações. Entre os resultados obtidos, houve uma redução significativa no tempo de obtenção das informações, de horas para minutos.

Palavras-chaves: análise de dados, análise de negócios, ETL, vendas.

\section{Introducción}

La información desempeña un papel decisivo en una organización, hasta el punto de convertirse en algunos casos en su principal patrimonio (Yao et al., 2010), ya que de esta depende el desarrollo de las actividades cotidianas y la posibilidad de alcanzar un alto nivel de competitividad y desarrollo. Es por esto que actualmente las empresas dedican gran parte de sus recursos a la obtención, procesamiento, aplicación y proyección de los datos con el fin de poder convertirlos en información que soporte la toma de decisiones. A esto se conoce como inteligencia de negocios y se espera, por tanto, que la información sea clara, precisa y adaptable a los requerimientos y perfiles de a quién va dirigida; además de ser rápida y estar disponible en el momento en que se necesite de una manera completa (Sharma et al., 2016).

El entorno empresarial es un sistema de complejidad creciente en el que permanentemente hay generación y ganancia de nueva información a partir de la interacción entre las organizaciones que lo componen (Ahmad, 2012). En el subsistema comercial de servicios en empresas del sector de IT esta interacción está basada principalmente en la relación con los clientes; sin embargo, influyen otras variables como cambios políticos, económicos, legales, así como el surgimiento de nuevas empresas con fuertes estrategias competitivas. Esto hace que elegir la mejor opción sea cada vez más complejo, sin mencionar los efectos de la globalización y el desarrollo de las tecnologías de la información, que son algunos de los factores que también influyen en el entorno (Hou, 2012).

Para el caso particular de la empresa de tecnología en estudio (con presencia a nivel latinoamericano) se realizan procesos manuales $y$ desgastantes para obtener los datos que pretenden pronosticar las ventas de cada periodo. Estos datos son entregados, al final del mes, por cada uno de los responsables en cada país para obtener el pronóstico total. Estas proyecciones repercuten directamente en la eficacia y eficiencia con que operan las áreas de producción, mercadotecnia, ventas, finanzas, personal, logística y otros departamentos funcionales de la compañía.

Del mismo modo, para los gerentes la importancia del pronóstico de ventas radica en entender el desempeño de la organización y si se deben tomar ciertas medidas en función de los datos obtenidos. El pronóstico de ventas permite planificar el crecimiento futuro, revisar la estrategia comercial y mantener la ventaja competitiva, desconocer su importancia puede traer graves consecuencias para el futuro de la empresa. El objetivo de este estudio fue proponer un modelo basado en datos históricos que permita agilizar el proceso de pronóstico y optimizar los resultados para la toma de decisiones.

La metodología aplicada se constituyó en tres fases: la primera consistió en un análisis de datos históricos de las ventas en el subsistema de servicios empresariales teniendo en cuenta dos periodos anuales. En la segunda fase se Ilevó a cabo la socialización y discusión de este análisis con un panel de expertos compuesto por cuatro gerentes de ventas. Finalmente, en la tercera se unificó la información recolectada en los pasos uno y dos para, posteriormente, realizar pruebas sobre una plataforma web. 
La herramienta utilizada permite la descarga, procesamiento y clasificación de los datos en las diferentes líneas de servicio del subsistema comercial. Se evidenció la reducción significativa del tiempo de obtención de la información de las ventas simplificando la labor. A su vez, la disminución de los costos y recursos asociados al incluir un método de pronóstico de ventas que genera importantes beneficios en comparación con la metodología previa.

\section{Inteligencia de negocios}

\section{Conceptos}

El concepto de inteligencia de negocios puede relacionarse con el concepto de éxito expresado en el libro El arte de la guerra de Sun Tzu. Para este autor, para alcanzarlo es imprescindible conocer tanto las fortalezas y debilidades de uno mismo, así como las del enemigo, ya que el desconocimiento de estas puede llevar a la derrota (Ahumada-Tello y Perusquia, 2016). Lo mismo ocurre en las organizaciones y en el ambiente empresarial, en donde los retos y las ventajas competitivas que se pueden lograr para alcanzar el éxito y la sostenibilidad en el mercado dependen en gran medida de obtener información significativa de los datos, diferenciando patrones y tendencias que permitan tomar decisiones más precisas (Dalla et al., 2018; Pozzaab et al., 2018).

La mejor forma de analizar el entorno no es el tratar de entenderlo como una colección de otros sistemas y organizaciones, sino como información a la cual la empresa debe acceder mediante actividades de investigación (Djatna y Munichputranto, 2015). No son los proveedores ni los clientes en sí mismos los que cuentan, sino la información disponible para la empresa acerca de sus metas, intereses, condiciones bajo las cuales se relacionan con la empresa y muchos otros aspectos de su comportamiento los que verdaderamente ayudan a identificar sus características y expectativas para con la organización (Safadi et al., 2017).
El uso de la inteligencia de negocios ha evolucionado a pasos agigantados en proporción al auge de las tecnologías de la información y al crecimiento de la industria global (Del et al., 2019). Son numerosas las definiciones del término inteligencia de negocios dadas por expertos en el área, pero suele definirse como la transformación de los datos de la compañía en conocimiento para obtener una ventaja competitiva (Gartner INC., 2019).

Se puede concluir que la inteligencia de negocios es el uso de tecnologías, procesos y herramientas para transformar los datos en información y la información en conocimiento, para que este último soporte y permita tomar decisiones más convenientes para que la empresa obtenga mejores resultados.

\section{Tecnologías existentes}

Por el nivel de confidencialidad de los datos a procesar, se determinó escoger entre las tres plataformas mejor calificadas desde el 2016 al 2019, según el cuadrante mágico de Gartner (figura 1). Esta organización año tras año lleva a cabo, mediante el estudio de casos aplicados y opiniones de expertos, un análisis de las mejores plataformas para inteligencia de negocios, basados en infraestructura, seguridad, gestión de datos, análisis y creación de contenidos, así como opciones para compartir contenidos y capacidades generales de la plataforma.

Dentro de las herramientas que lideran el mercado encontramos Tableu, una herramienta que cuenta con la mayor variedad de opciones en cuanto a gráficos y análisis de datos, lo que brinda diversidad y una alta tasa de crecimiento en su ambiente de desarrollo al enfocarse en la experiencia del cliente. Su precio es algo elevado con respecto a sus competidores, además carece de compatibilidad con modelos de datos complejos (Tableau, 2019).

Por otro lado, Qlik View es una herramienta desarrollada por Qlik, la cual se caracteriza principalmente por la facilidad en el procesamiento 


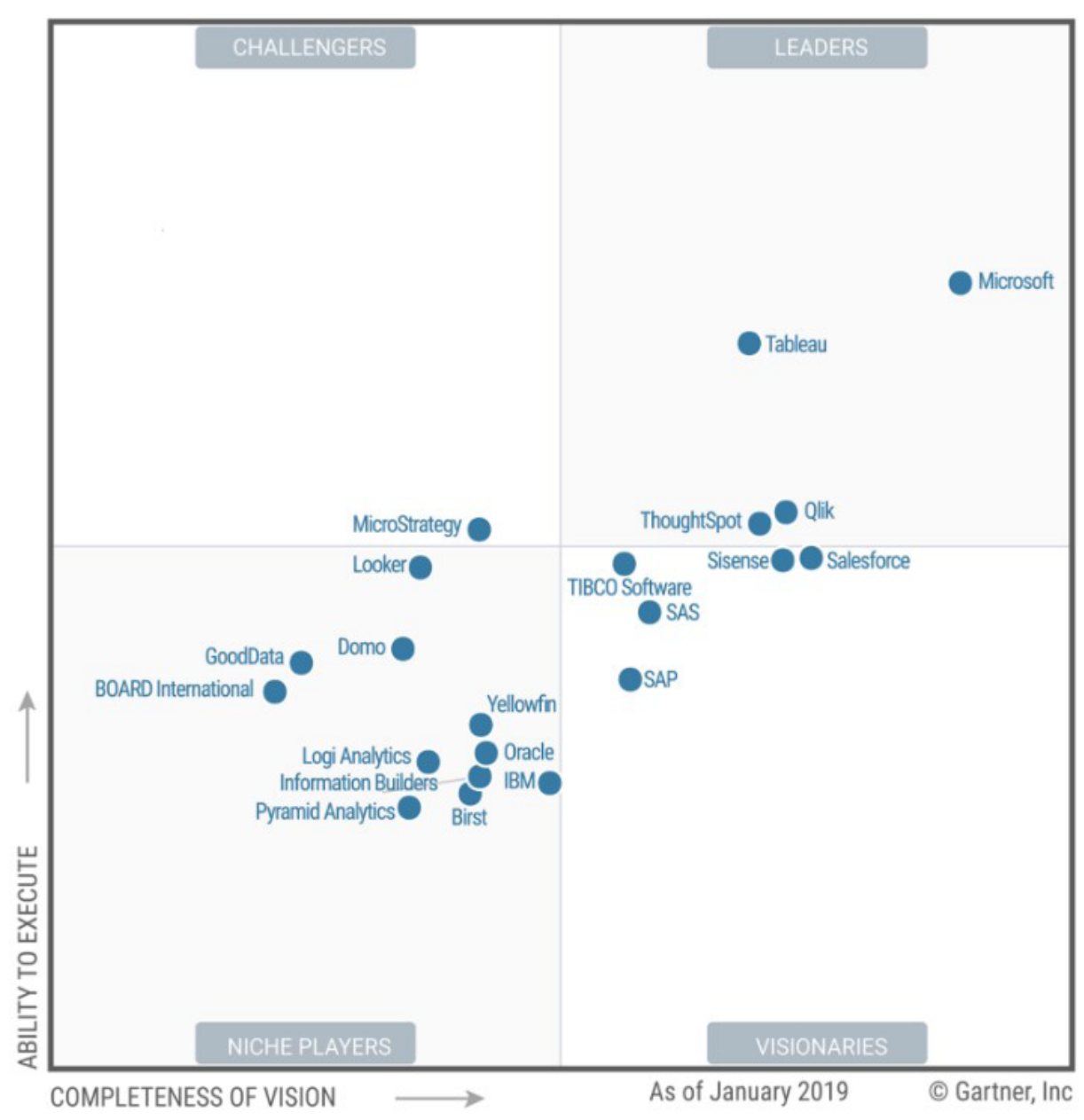

Figura 1. Cuadrante mágico de Gartner 2019.

Fuente: Gartner INC (2019).

de datos con modelos robustos, cuenta con una gran red de socios que contribuyen en su desarrollo, con lo cual ofrece gran diversidad de herramientas. Fue de las primeras herramientas en ofrecer un entorno de tableros y modelos interactivos, en contraste a esto, debido a sus altos costos, presentan poco crecimiento, además su compatibilidad con bases de datos de terceros es limitada (Qlik, 2019).

Finalmente, Power $\mathrm{Bl}$ es la herramienta desarrollada por Microsoft que se encuentra en lo más alto de la tabla clasificatoria con características atractivas gracias a su facilidad de uso y el atractivo visual que logran sus reportes para el usuario final, generando una excelente experiencia de uso. Tiene como ventajas el bajo costo en su licenciamiento y su compatibilidad con múltiples fuentes en la nube, pero lastimosamente solo es posible realizar conexiones ágiles y de autoservicio con su nube en Azure, más no con bases de datos en SQL, para ello se debe recurrir a software de terceros (Microsoft, 2019). Por sus características esta es la opción seleccionada para el modelo propuesto.

\section{Los gerentes como tomadores de decisiones}

La toma de decisiones es la esencia del trabajo del gerente, de modo que la calidad de sus decisiones tiene gran influencia en el éxito o fracaso de una organización (Vera-Baquero et al., 2014). A ello debemos sumarle que a diario se enfrentan a 
limitaciones como la cultura de la organización, el medio externo, políticas internas y consideraciones de poder (Kim et al., 2016).

Todos los gerentes toman decisiones que pueden abarcar temas relacionados a la supervivencia de la empresa hasta el cierre de un contrato de compra de insumos o el ascenso de un empleado. Así las cosas, el futuro de una empresa depende entonces de las decisiones que tomen sus gerentes para responder a las condiciones del mercado y de la competencia (Piontkewicz et al., 2016).

\section{Metodología}

El modelo para la toma de decisiones se desarrolló mediante tres fases: la primera consistió en un análisis de datos históricos de las ventas en el subsistema de servicios empresariales teniendo en cuenta dos periodos anuales. En la segunda fase se llevó a cabo la socialización y discusión de este análisis con un panel de expertos compuesto por cuatro gerentes de ventas de diferentes líneas de negocio en Latinoamérica. Finalmente, en la tercera fase se unificó la información recolectada en los pasos uno y dos, para a continuación llevar a cabo pruebas sobre una plataforma web (figura 2).

El modelo se alimenta de los datos referentes a negocios activos en el tercer año los cuales se encuentran en una plataforma en la nube y brinda al tomador de decisiones tres escenarios estimados para el cierre del periodo, estos son:

- Peor de los casos: en este escenario se encuentran los negocios de más alta probabilidad de cierre.

- Más probable: en este escenario se tienen en cuenta los negocios del primer escenario y los negocios con una probabilidad media.

- Mejor de los casos: este es el escenario optimista y se tienen en cuenta los dos primeros escenarios y los negocios de baja probabilidad.

En el mundo de ventas la persona encargada debe llevar a cabo seguimiento sobre los negocios

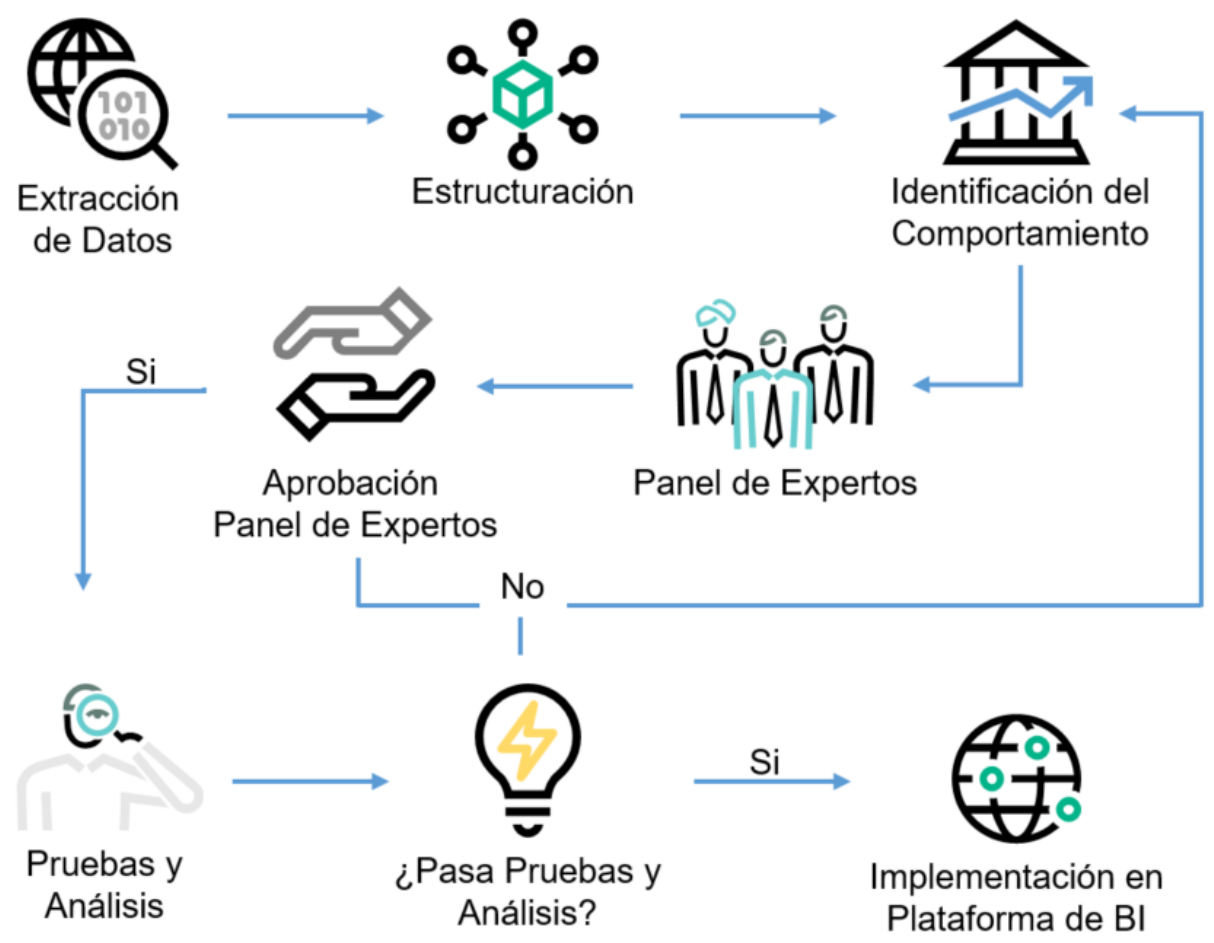

Figura 2. Metodología utilizada.

Fuente: elaboración propia de los autores. 
que se presentan con diferentes clientes teniendo en cuenta parámetros para así determinar con facilidad el avance de cada negocio (Agnihotri et al., 2017). Dichos parámetros están representados por los criterios y las etapas del ciclo de ventas. Los criterios hacen referencia al estado del negocio basado en las interacciones con el cliente y los riesgos tanto internos como externos. Estos criterios los define el vendedor según su experiencia con cada tipo de cliente o por el tipo de solución que se esté ofreciendo; además, son de alto impacto pues según como se clasifiquen se tienen o no en cuenta los negocios. Para el pronóstico se categorizaron cinco criterios, los cuales son:

- Pipeline: es el criterio inicial cuando se crea el negocio, en el cual hasta el momento se están haciendo la validación de datos y el tipo de negocio que se va a desarrollar; estos negocios se encuentran por fuera del pronóstico del periodo evaluado.

- Upside: son negocios con algún avance, que no tienen garantía de cerrarse en el periodo en curso, pero que podrían darse si sale todo según lo planeado; estos negocios se encuentran por fuera del pronóstico del periodo evaluado.

- Commit: estos negocios se encuentran en su etapa final y se cerraran en el periodo en curso, por lo cual se encuentran en el pronóstico del periodo evaluado.

- Won: son negocios de los cuales ya se recibió orden de compra por parte del cliente, por lo cual se encuentran en el pronóstico del periodo evaluado.

- Omitted: técnicamente este no es un criterio, pero es la forma de identificar los negocios que se perdieron por alguna razón.

En cuanto a las etapas del ciclo de ventas, estas permiten definir el estado de un negocio en un determinado periodo o momento. Dependen de la interacción entre el cliente y el vendedor con su equipo de soporte. Este proceso se representa por un embudo, pues conforme avanza se van decantando los clientes que tienen real interés y recursos para hacer la compra. Las etapas definidas incluyen:

- Entendimiento del cliente: en esta etapa se busca entender las necesidades del cliente.

- Validación del negocio: en esta etapa se valida que se tiene la solución a las necesidades del cliente.

- Calificación del negocio: en esta etapa se determina la posible o posibles soluciones para satisfacer las necesidades del cliente.

- Desarrollo de la solución: en esta etapa el equipo de soporte de ventas desarrolla una solución acorde a las necesidades del cliente.

- Presentación de la solución: en esta etapa se presenta de manera formal la solución propuesta para que el cliente apruebe o pida cambios sobre esta.

- Negociación y cierre: en esta etapa se lleva a cabo la negociación final para fijar los términos económicos y legales.

- Ganado en desarrollo y entrega: en esta etapa se realiza la entrega y el desarrollo de la solución.

Por la sensibilidad de los datos y el comportamiento único de la información, se tomó como pronóstico la combinación de escenarios generados por la interacción entre los criterios y las etapas del ciclo de ventas, método que se comparó frente a la información histórica obtenida en el tercer año.

\section{Resultados}

Tras obtener la interacción óptima entre los criterios y las etapas del ciclo de ventas, se generó un modelo el cual se sometió a pruebas, en el cual se alimentó con datos correspondientes a las ventas durante dos periodos anuales y se comparó contra datos de pronóstico reales trimestrales en ese mismo periodo.

En la tabla 1 se muestran los resultados mensuales de cada escenario frente al cierre real de cada periodo. Las diferencias porcentuales del 
escenario esperado frente al cierre real son mayores al $15 \%$, en apenas 5 meses; esto se debió a calidad de la información, pues en ocasiones los vendedores no reportan el valor real de los negocios sino hasta su cierre o por una mala evaluación de este frente al negocio.

El modelo se desarrolló de tal forma que el escenario esperado brinde un pronóstico estimado para el periodo en evaluación y los escenarios peor de los casos y mejor de los casos sirvan de limites inferior y superior, respectivamente. La robustez del modelo está respaldada por la fuente de la información que lo alimenta.

A continuación, se planteó una prueba de hipótesis de diferencia de medias para los datos obtenidos con el modelo para toma de decisiones, contra los datos reales para los años 1 y 2. Se definió un alpha de 0,05 para determinar si se acepta o no que los datos reales tienen diferencia con los datos calculados para el escenario esperado.
H0: $\mu$ real $=\mu$ calculado

$$
\text { H1: } \mu \text { real } \neq \mu \text { calculado }
$$

Se realizó Anova de un solo factor, con la cual se obtienen los siguientes resultados:

$$
\begin{aligned}
& \boldsymbol{F}=1,067 \\
& \boldsymbol{P}-\text { Value }=0,307 \\
& \text { F. Crit }=4,052
\end{aligned}
$$

Observamos que el $p$-Value es de 0,307, mayor que el alpha de 0,05; por lo tanto, se acepta $\mathrm{H} 0$, lo cual nos indica que existe suficiente evidencia estadística para aceptar que la media de los datos reales es igual a la media de los datos calculados.

\begin{tabular}{|c|c|c|c|c|c|}
\hline Año & Periodo & Mes & $\begin{array}{c}\text { Peor de los } \\
\text { casos }(\%)\end{array}$ & $\begin{array}{c}\text { E sperado } \\
(\%)\end{array}$ & $\begin{array}{c}\text { Mejor de los } \\
\text { casos }(\%)\end{array}$ \\
\hline \multirow{12}{*}{ - } & \multirow[t]{3}{*}{ Q1 } & $M 1$ & -36 & -3 & 29 \\
\hline & & M2 & -33 & -6 & 12 \\
\hline & & M3 & -11 & 5 & 7 \\
\hline & \multirow[t]{3}{*}{ Q2 } & M1 & -16 & 12 & 45 \\
\hline & & M2 & -34 & -6 & 19 \\
\hline & & M3 & 9 & 32 & 33 \\
\hline & \multirow[t]{3}{*}{ Q3 } & M1 & -26 & 2 & 29 \\
\hline & & M2 & -37 & -5 & 18 \\
\hline & & M3 & -20 & 0 & 2 \\
\hline & \multirow[t]{3}{*}{ Q4 } & M1 & -13 & 20 & 63 \\
\hline & & M2 & -30 & 3 & 35 \\
\hline & & M3 & -18 & 4 & 7 \\
\hline \multirow{12}{*}{$N$} & \multirow[t]{3}{*}{ Q1 } & M1 & -28 & 1 & 29 \\
\hline & & M2 & -32 & -12 & 10 \\
\hline & & M3 & -21 & -8 & -5 \\
\hline & \multirow[t]{3}{*}{ Q2 } & M1 & -34 & -6 & 21 \\
\hline & & M2 & -42 & -15 & 8 \\
\hline & & M3 & -26 & 0 & 1 \\
\hline & \multirow[t]{3}{*}{ Q3 } & M1 & -40 & -14 & -4 \\
\hline & & M2 & -56 & -9 & 10 \\
\hline & & M3 & -59 & -32 & -31 \\
\hline & \multirow[t]{3}{*}{ Q4 } & M1 & -38 & -11 & 8 \\
\hline & & M2 & -46 & -18 & 14 \\
\hline & & M3 & -24 & -4 & -2 \\
\hline
\end{tabular}
Así las cosas, podemos concluir que el modelo de pronóstico es aplicable.

Tabla 1. Resultados modelo toma de decisiones, años 1 y 2.

Fuente: elaboración propia de los autores. 
Tabla 2. Calculado vs. resultados 2018 (\%).

\begin{tabular}{ccccc}
\hline Período & Mes & Peor de los casos (\%) & Esperado (\%) & Mejor de los casos (\%) \\
& 1 & -63 & -10 & 2 \\
Q1 & 2 & -59 & -40 & 7 \\
& 3 & -16 & -2 & 0 \\
Q2 & 1 & -31 & 16 & 27 \\
& 2 & -20 & 0 & 30 \\
& 3 & -2 & 7 & 3 \\
& 1 & -49 & -16 & 15 \\
& 2 & -29 & -12 & -3 \\
& 3 & -13 & 4 & 7 \\
& 1 & -15 & -5 & 10 \\
\hline
\end{tabular}

Fuente: elaboración propia de los autores.

También se evaluaron los resultados obtenidos en el escenario esperado con la información del año 3 (tabla 2), mediante una prueba de hipótesis de diferencia de medias con un alpha de 0,05 para determinar si se acepta o no que los datos reales tienen diferencia con los datos calculados para dicho escenario.

$$
\begin{aligned}
& \text { H0: } \mu \text { real }=\mu \text { calculado } \\
& \text { H1: } \mu \text { real } \neq \mu \text { calculado }
\end{aligned}
$$

Se realiza Anova de un solo factor con la cual se obtienen los siguientes resultados:

$$
\begin{aligned}
& \boldsymbol{F}=1,833 \\
& P-\text { Value }=0,195 \\
& \text { F. Crit }=4,494
\end{aligned}
$$

Observamos que el $p$-Value es de 0,195 mayor que el alpha de 0,05; por lo tanto, se acepta H0, lo cual nos indica que existe suficiente evidencia estadística para aceptar que la media de los datos reales es igual a la media de los datos calculados. Por lo anterior, podemos concluir que el modelo de pronóstico es aplicable. Adicionalmente, se evidencia en la tabla 2 que los escenarios peor de los casos y mejor de los casos muestran resultados por encima y por debajo, respectivamente, del número oficial de cierre para cada periodo; por lo que se mantiene la similitud a líneas de control en las cuales se busca que el resultado final no esté por fuera de estas.

\section{Conclusiones}

El uso de herramientas de inteligencia de negocios en combinación con un modelo para la toma de decisiones brinda a las empresas ventajas competitivas, tales como el tiempo de obtención y la precisión de la información. Durante la investigación se evidenció una mejora con respecto al método utilizado normalmente. Antes, para la realización del pronóstico, se usaba Microsoft Excel, seleccionando de forma manual los negocios que suministraban la información para el pronóstico del periodo y esto podía tomar alrededor de seis horas.

Al aplicar, mediante el uso de Microsoft Power $\mathrm{BI}$, un modelo para la toma de decisiones relacionadas con las ventas (que se alimenta directamente de la plataforma) se generan opciones precisas, de forma automática, para el pronóstico del periodo que corresponda. La principal ventaja en el uso de esta herramienta es el tiempo en el cual se obtiene la información, pues este se estima en apenas seis a diez minutos, dependiendo de la conexión de internet del usuario. 
Este modelo tiene una limitación importante: la calidad y la veracidad de la información cargada en la plataforma de ventas dependen de los vendedores, ya que ellos son encargados de subirla y actualizarla. Para contribuir a mitigar información ruidosa en el modelo se llevan cabo campañas de limpieza que aporten a mejorar la calidad.

Cabe aclarar que, durante la investigación, el modelo para la toma de decisiones no discriminó las líneas de negocio, por lo cual es posible encontrar diferentes tendencias dependiendo de la solución ofrecida. Tampoco se hizo una clasificación por clientes, teniendo en cuenta que hay clientes grandes, medianos y pequeños, los cuales tienen diferentes comportamientos en sus hábitos de compras.

Este artículo aporta al conocimiento al evidenciar una aplicación práctica y económica para pronosticar las ventas en una empresa del sector tecnológico sin recurrir a soluciones demasiado robustas o fuera del alcance. También permite ver cómo grandes empresas multinacionales con muchas fortalezas se enfrentan a este tipo de retos diariamente descuidando o desconociendo soluciones prácticas y eficientes.

\section{Referencias}

Agnihotri, R., Trainor, K. J., Itani, O. S., Rodríguez, M. (2017). Examining the role of sales-based CRM technology and social media use on post-sale service behaviors in India. Journal of Business Research, 81, 144-154. https://doi.org/10.1016/j.jbusres.2017.08.021

Ahmad, M. S. (2012). Impact of Organizational Culture on Performance Management Practices in Pakistan. Business Intelligence Journal, 5(1), 50-55.

Ahumada Tello, V. E., Perusquia, J. M. A. (2016). Inteligencia de negocios: estrategia para el desarrollo de competitividad en empresas de base tecnológica. Contaduría y Administración, 61(1), 127-158. https://doi.org/https://doi.org/10.1016/j.cya.2015.09.006
Dalla, I., Goetz, O., Michel, J. (2018). Implementation e ff ects in the relationship between CRM and its performance. Journal of Business Research, 89, 391-403. https://doi.org/10.1016/j. jbusres.2018.02.004

Del, M., Giannone, D., Giannoni, M. P., Tambalotti, A. (2019). Global trends in interest rates. Journal of International Economics, 118, 248-262. https://doi.org/10.1016/j. jinteco.2019.01.010

Djatna, T., Munichputranto, F. (2015). An analysis and design of mobile business intelligence system for productivity measurement and evaluation in tire curing production line. Procedia Manufacturing, 4, 438-444. https://doi. org/10.1016/j.promfg.2015.11.060

Gartner INC. (2019). The 2019 Analytics and BI Magic Quadrant Highlights.

Hou, C. K. (2012). Examining the effect of user satisfaction on system usage and individual performance with business intelligence systems: An empirical study of Taiwan's electronics industry. International Journal of Information Management, 32(6), 560-573. https://doi.org/10.1016/j.ijinfomgt.2012.03.001

Kim, A., Khon, N., Aidosova, Z. (2016). Emotional intelligence of a coach as a factor of coach-student interaction. Procedia-Social and Behavioral Sciences, 236, 265-270. https://doi. org/10.1016/j.sbspro.2016.12.026

Microsoft (2019). Power BI | Herramientas de BI para la visualización de datos interactivos.

Piontkewicz, R., Freitas, M. D. C. D., Kemczinski, A., Martin, C. D. S. (2016). Management of intellectual capital in a system of management accounting information. 6th International Conference on Computers Communications and Control, ICCCC 2016, 180-187. https://doi. org/10.1109/ICCCC.2016.7496758

Pozzaab, I., Goetzc, O., Sahutd, J. M. (2018). Implementation effects in the relationship between CRM and its performance. Journal of Business Research, 89, 391-403. https://doi.org/10.1016/j.jbusres.2018.02.004 
Qlik (2019). QlikView-Visual Intelligence.

Safadi, M., Ma, J., Wickramasuriya, R., Daly, D., Perez, P., Kokogiannakisb, G. (2017). Mapping for the future : Business intelligence tool to map regional housing stock. Procedia Engineering, 180, 1684-1694. https://doi.org/10.1016/j.proeng.2017.04.331

Sharma, M., Singh, G., Singh, R. (2016). Design and analysis of stochastic DSS query optimizers in a distributed database system. Egyptian Informatics Journal, 17(2), 161-173. https://doi.org/10.1016/j.eij.2015.10.003
Tableau (2019). Información | Tableau Software. Vera-Baquero, A., Colomo-Palacios, R., Molloy, O. (2014). Towards a Process to Guide Big Data Based Decision Support Systems for Business Processes. Procedia Technology, 16(2212), 11-21. https://doi.org/10.1016/j.protcy.2014.10.063 Yao, Z., Eklund, T., Back, B. (2010). Using SOMWard Clustering and predictive analytics for conducting customer segmentation. Proceedings-IEEE International Conference on Data Mining, ICDM, 639-646. https://doi. org/10.1109/ICDMW.2010.121 